\title{
Transfer reactions as tool for nuclear astrophysics: the ${ }^{13} \mathbf{C}(\alpha, \mathbf{n}){ }^{16} \mathbf{O}$ case
}

\author{
F. Hammache ${ }^{1 *}$, M. G. Pellegriti ${ }^{1}$ P. Rousse $^{1}$, L.Audouin ${ }^{1}$, D. Beaumel ${ }^{1}$, P. $^{2}$ \\ Descouvemont $^{2}$, S. Fortier ${ }^{1}$, L. Gaudefroy ${ }^{3}$, J. Kiener ${ }^{4}$, A. Lefebvre-Schuhl ${ }^{4}$, M. \\ Stanoiu ${ }^{5}$, V. Tatischeff ${ }^{4}$ \\ 1 IPN-Orsay, IN2P3-Université Paris XI, F-91406 Orsay Cedex, France \\ 2 Physique Nucléaire Théorique et Physique Mathématique, ULB CP229, B-1050 Brussels, \\ Belgium \\ 3 GANIL, IN2P3-CNRS, Caen, France \\ 4 CSNSM, IN2P3-Université Paris XI, F-91405 Orsay Cedex, France \\ 5 GSI,Postfach 110552, D-64220 Darmstadt, Germany \\ E-mail: hammachedipno.in2p3.fr
}

The cross section of the nuclear reactions involved in stellar nucleosynthesis (e.g. AGB stars) are often very difficult to measure directly at stellar energies because of their very small value. Moreover, this situation can be complicated by the existence of very low energy resonances and/or subthreshold resonances. Indirect methods such as transfer reactions and the ANC method offer the possibility to overcome these difficulties. In this context, recent indirect measurement of the reaction ${ }^{13} \mathrm{C}(\alpha, \mathrm{n}){ }^{16} \mathrm{O}$ is presented.

The ${ }^{13} \mathrm{C}(\alpha, \mathrm{n}){ }^{16} \mathrm{O}$ reaction is considered as the main neutron source for s-process in low-mass asymptotic giant branch stars. At low energies of astrophysical interest, the contribution of the subthreshold state $6.356 \mathrm{MeV}$ of ${ }^{17} \mathrm{O}$ to the ${ }^{13} \mathrm{C}(\alpha, \mathrm{n}){ }^{16} \mathrm{O}$ cross section should be taken into account. However, the results of previous studies of this contribution lead to different conclusions. Hence, we investigated the effect of this resonance on the astrophysical S-factor through a new precise measurement of the alpha spectroscopic factor, $\mathrm{S}_{\alpha}$, and the corresponding ANC of the 6.356 MeV state using the transfer reaction ${ }^{13} \mathrm{C}\left({ }^{7} \mathrm{Li}, \mathrm{t}\right){ }^{17} \mathrm{O}$ at two different incident energies. The measured angular distributions and the obtained spectroscopic factor and the asymptotic normalization constant (ANC) are presented as well as their impact on ${ }^{13} \mathrm{C}(\alpha, n){ }^{16} \mathrm{O}$ cross section and reaction rate.

10th Symposium on Nuclei in the Cosmos

July 27 - August 12008

Mackinac Island, Michigan, USA

\footnotetext{
* Speaker.

†Present address: Dipartimento di Fisica e Astronomia, Universitá di Catania and Laboratori Nazionali del Sud INFN, Catania, Italy
} 


\section{Introduction}

Nearly half of the heavy elements observed in the Universe are produced by a slow sequence of neutron capture reactions, the so-called s-process. For this astrophysical process, two neutron sources are used in all evolution models: the reactions ${ }^{13} \mathrm{C}(\alpha, \mathrm{n}){ }^{16} \mathrm{O}$ and ${ }^{22} \mathrm{Ne}(\alpha, \mathrm{n}){ }^{25} \mathrm{Mg}$. For the AGB stars of 1-3 solar masses at low temperatures, only the ${ }^{13} \mathrm{C}(\alpha, \mathrm{n}){ }^{16} \mathrm{O}$ reaction is considered as the main neutron source for the s-process $[1,2,3]$. Hence, all the models describing the s-process nucleosynthesis in these AGB stars depend on the neutron flux from the ${ }^{13} \mathrm{C}(\alpha, \mathrm{n})^{16} \mathrm{O}$ reaction and so on the cross section of this reaction which occurs in these stars at temperatures around $10^{8} \mathrm{~K}$, i.e around the Gamow peak $\mathrm{E}_{c m} \sim 190 \mathrm{keV}$.

Direct measurement of ${ }^{13} \mathrm{C}(\alpha, \mathrm{n}){ }^{16} \mathrm{O}$ reaction at this energy is extremely difficult because the cross section decreases drastically when the incident $\alpha$ energy decreases. Thus, direct measurements [4] have only been performed down to $270 \mathrm{keV}$ (see Fig. 1) too far away from the energy range of interest around $190 \mathrm{keV}$. R-matrix extrapolations [5, 6] of the cross sections measured at higher energies have then to be performed and have to include the contribution of the $6.356 \mathrm{MeV}$ state of the compound nucleus ${ }^{17} \mathrm{O}$ which lies $3 \mathrm{keV}$ below the $\alpha+{ }^{13} \mathrm{C}$ threshold. This contribution strongly depends on the $\alpha$-spectroscopic factor $\mathrm{S}_{\alpha}$ of this state. R-matrix extrapolations [7], which consider a large value of 0.7 for the $\alpha$ spectroscopic factor $\left(\mathrm{S}_{\alpha}\right)$ of the subthreshold state at 6.356 $\mathrm{MeV}$ lead to a rapid increase of the S-factor when the energy decreases to zero (see curve A in Fig. 1 ), while they lead to constant extrapolated $S$-factor at low energies when they use a null $S_{\alpha}$ (see curve B in Fig.1).

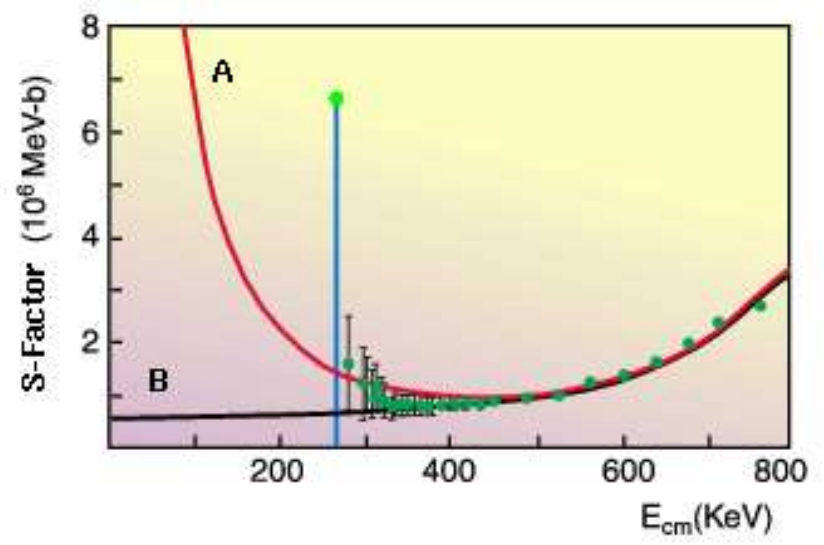

Figure 1: ${ }^{13} \mathrm{C}(\alpha, \mathrm{n}){ }^{16} \mathrm{O}$ astrophysical S-factors as a function of center of mass energy. The data points are the direct measurements of Drotleff et al. [4]. Curves A and B inferred from R-matrix calculations using an $\alpha$ spectroscopic factor for the subthreshold state at $6.356 \mathrm{MeV}$ of about 0.7 and 0 respectively.

If the experimental points in Fig.1 suggest a rise of the S-factor when the energy decreases as expected with the usually used spectroscopic factors $[5,6]$ in the s-models $\left(S_{\alpha} \sim 0.3-0.7\right.$ (curve A)), the error bars are too large to well discriminate curves A and B.

Kubono et al. [8] then suggested to measure the $S_{\alpha}$ of the subthreshold state at $6.356 \mathrm{MeV}$ via ${ }^{13} \mathrm{C}\left({ }^{6} \mathrm{Li}, \mathrm{d}\right){ }^{17} \mathrm{O} \alpha$ transfer reaction at the laboratory energy of $60 \mathrm{MeV}$. 
Through a finite-range DWBA analysis of their transfer angular distributions, a very small value of $\mathrm{S}_{\alpha} \sim 0.01$ was deduced for the $6.356 \mathrm{MeV} 1 / 2^{+}$state of ${ }^{17} \mathrm{O}$. This value leads to a very small contribution of this subthreshold state to the cross section of ${ }^{13} \mathrm{C}(\alpha, \mathrm{n}){ }^{16} \mathrm{O}$ at energies of astrophysical interest and thus to the S-factor. This result has dramatic effects on the neutron flux and subsequently on the onset of the s-process nucleosynthesis. However, a reanalysis of the same data by Keeley et al. [9] ends up to a drastically different conclusion since they found a value of $\mathrm{S}_{\alpha}$ of about 0.4 , forty times higher than Kubono's value and consistent with the values $\left(\mathrm{S}_{\alpha} \sim 0.3\right.$ 0.7) usually used in astrophysical calculations. In addition to these controversial results, the new determination of the contribution of the $1 / 2^{+}$state to the total ${ }^{13} \mathrm{C}(\alpha, \mathrm{n}){ }^{16} \mathrm{O}$ astrophysical S-factor, by the recent ${ }^{13} \mathrm{C}\left({ }^{6} \mathrm{Li}, \mathrm{d}\right){ }^{17} \mathrm{O}$ ANC measurement [10], was found 10 times smaller than Nacre [7] adopted value (based on $\mathrm{S}_{\alpha}=0.1-0.7$ ) but 5 times larger than the result of Kubono et al. at the energy of $190 \mathrm{keV}$.

Therefore, it appeared highly desirable to perform a new precise determination of this $\alpha$ spectroscopic factor through another transfer reaction ${ }^{13} \mathrm{C}\left({ }^{7} \mathrm{Li}, \mathrm{t}\right){ }^{17} \mathrm{O}$ and an improved DWBA analysis.

\section{Experiment description}

The experiment was performed using a ${ }^{7} \mathrm{Li}^{3+}$ beam provided by the Orsay TANDEM. Two self-supporting enriched ${ }^{13} \mathrm{C}$ targets, with a ${ }^{13} \mathrm{C}$ thickness of $0.072 \pm 0.004 \mathrm{mg} / \mathrm{cm}^{2}$ and $0.133 \pm$ $0.007 \mathrm{mg} / \mathrm{cm}^{2}$ and an initial purity of about $90 \%$ were used. A ${ }^{12} \mathrm{C}$ target of $0.080 \pm 0.004 \mathrm{mg} / \mathrm{cm}^{2}$ was also used for calibration purposes and background subtraction. The absolute amount of ${ }^{13} \mathrm{C}$ and the final amount of ${ }^{12} \mathrm{C}$ in the ${ }^{13} \mathrm{C}$ target were deduced, respectively, from an $\alpha$-energy loss measurement and by comparing the measured ratio between $28 \mathrm{MeV}$ elastically scattered particles at $21^{\circ}$ from the ${ }^{12} \mathrm{C}$ nuclei in ${ }^{12} \mathrm{C}$ and ${ }^{13} \mathrm{C}$ targets. The reaction products were analyzed with an Enge Split-pole magnetic spectrometer and detected at the focal plane by a $50 \mathrm{~cm}$ long positionsensitive gas chamber and a $\Delta \mathrm{E}$ proportional gas-counter. The particle identification was made unambiguously using $\Delta \mathrm{E}$ versus position measurements.

The tritons were detected at angles ranging from 0 to 31 degrees corresponding to angles up to 43 degrees in the center of mass frame. The beam and ${ }^{12} \mathrm{C}$ amount were continuously monitored with a telescope of silicon detectors mounted inside the scattering chamber at $\theta_{\text {lab }}=35^{\circ}$.

Due to the presence of ${ }^{12} \mathrm{C}$ impurities, spectra coming from the ${ }^{13} \mathrm{C}\left({ }^{7} \mathrm{Li}, \mathrm{t}\right){ }^{17} \mathrm{O}$ reaction were contaminated by the excited levels of ${ }^{16} \mathrm{O}$, produced via the reaction ${ }^{12} \mathrm{C}\left({ }^{7} \mathrm{Li}, \mathrm{t}\right){ }^{16} \mathrm{O}$. So the $\left({ }^{7} \mathrm{Li}, \mathrm{t}\right)$ reaction was measured on both ${ }^{13} \mathrm{C}$ and ${ }^{12} \mathrm{C}$ targets at each angle with the same setup.

\section{Results}

The experimental ${ }^{13} \mathrm{C}\left({ }^{7} \mathrm{Li}, \mathrm{t}\right){ }^{17} \mathrm{O}$ differential cross sections measured for the 6.356, 3.055, 4.55 and $7.38 \mathrm{MeV}$, at the two incident energies of 34 and $28 \mathrm{MeV}$, are displayed in Fig.2a and Fig.2b, respectively. In the $34 \mathrm{MeV}$ column, the $3.055 \mathrm{MeV}$ data are Clark's measurements [11] at 35.5 $\mathrm{MeV}$. The accuracy assigned to our measured cross sections includes the uncertainties on the peak yield, the number of target atoms, the solid angle and the integrated charge except for the zero degree run (no charge measurement) where the number of counts in the silicon monitor detector placed at $35^{\circ}$ was used. 
Calculations with finite-range DWBA method, using the FRESCO code [12], were performed. For the triton channel, the optical model potentials used were taken from Garrett et al. [13] (POT I-f7/2 shell). Concerning the ${ }^{7} \mathrm{Li}$ channel, we used for the transfer data at $34 \mathrm{MeV}$, the optical potential parameters of Schumacher et al. [14] who performed ${ }^{7} \mathrm{Li}$ elastic scattering measurements on ${ }^{13} \mathrm{C}$ at the same energy. Concerning the data at $28 \mathrm{MeV}$, we used both those from Schumacher et al. as well as those provided from fitting our elastic scattering cross sections. The optical potential parameters finally selected are those giving the best fit for all the studied transitions in the $\left({ }^{7} \mathrm{Li}, \mathrm{t}\right)$ reaction.
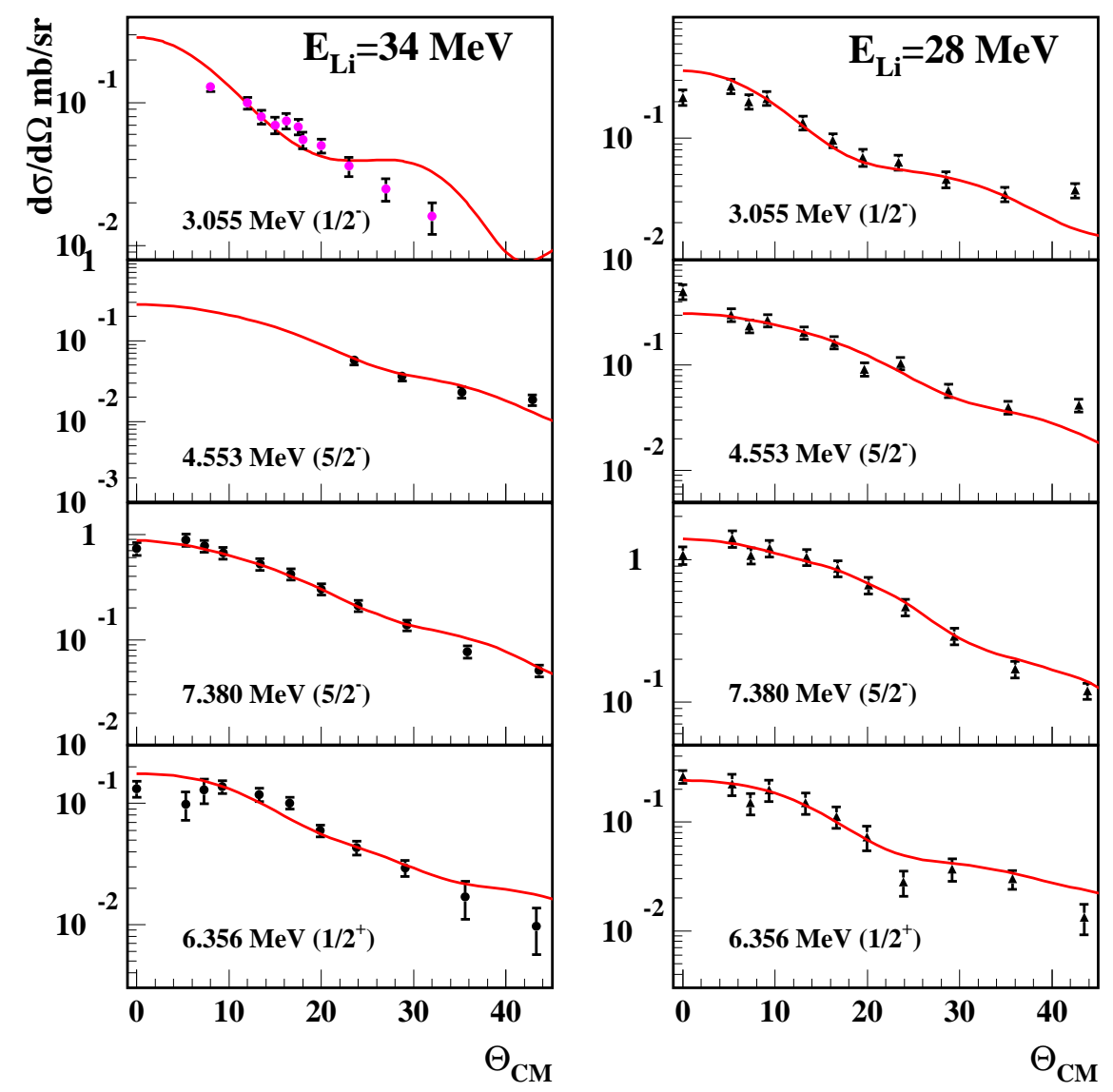

Figure 2: Experimental differential cross sections of the ${ }^{13} \mathrm{C}\left({ }^{7} \mathrm{Li}, \mathrm{t}\right){ }^{17} \mathrm{O}$ reaction obtained at 28 and $34 \mathrm{MeV}$, compared with finite-range DWBA calculations normalized to the data.

For the $\alpha$ wave function in ${ }^{17} \mathrm{O}$, an $\alpha+{ }^{13} \mathrm{C}$ Wood-Saxon potential was used. A range of radius (3.6 fm $\leq \mathrm{R} \leq 4.2 \mathrm{fm}$ ) and diffuseness ( $0.61 \mathrm{fm} \leq \mathrm{a} \leq 0.76 \mathrm{fm}$ ) was selected by using the maximum likelihood function (set at $3 \sigma$ level) on both 6.356 and $3.055 \mathrm{MeV}$ distributions (that's why the selected zone is so narrow). Within this radius and diffuseness range, the boundary values $\mathrm{R}=3.6 \mathrm{fm}$ and $\mathrm{a}=0.76 \mathrm{fm}$ provide the best fit for the angular distributions of the four studied states at both incident energies (fig.2).

The depth was adjusted to reproduce the binding energy of each considered ${ }^{17} \mathrm{O}$ state and 
the number of radial nodes $\mathrm{N}$ was fixed by the most probable configuration ( $\mathrm{N}$ (Fresco) $=4$ for 6.356 $\mathrm{MeV}$ ) coming from the oscillator energy conservation relation. The calculated angular distributions normalized to the data are shown in Figure 2. For both incident energies, the calculated curves agree fairly well with all the measured angular distributions of the different populated states. The $\alpha$-spectroscopic factors were extracted from the normalization of the finite-range DWBA curves to the experimental data, $\mathrm{S}_{\alpha}=\frac{\sigma_{e x p}}{\sigma_{D W}}$. The spectroscopic factor for the overlap between $\alpha+\mathrm{t}$ and ${ }^{7} \mathrm{Li}$ was taken to be 1.0 [15]. With this value, the $\alpha$-spectroscopic factor of the reference state at 3.055 $\mathrm{MeV}$ is found to be $\mathrm{S}_{\alpha}=0.26 \pm 0.06$, similar to that obtained in nuclear model calculations $\mathrm{S}_{\alpha}=0.25$ [16] . A close value was obtained by Keeley et al. [9].

The good agreement between the DWBA calculations and the measured differential cross sections of the different excited states of ${ }^{17} \mathrm{O}$ at the two bombarding energies of $28 \mathrm{MeV}$ and $34 \mathrm{MeV}$ respectively, gives strong evidence of the direct nature of the $\left({ }^{7} \mathrm{Li}, \mathrm{t}\right)$ reaction populating these levels and confidence in our DWBA analysis. An $S_{\alpha}$ mean value of $0.29 \pm 0.11$ is deduced for the state of interest at $6.356 \mathrm{MeV}$ of ${ }^{17} \mathrm{O}$, which is in good agreement with that obtained by Keeley et al. [9] and those used earlier $\left(\mathrm{S}_{\alpha} \approx 0.3-0.7\right)$ in the s-process models. The uncertainty on the extracted $\alpha$ spectroscopic factor for our state of interest $(6.356 \mathrm{MeV})$ was evaluated from the dispersion of the deduced $\mathrm{S}_{\alpha}$ values at the two incident energies and using different sets of optical potentials in the entrance [14] and exit channels [13] and different $\alpha-{ }^{13} \mathrm{C}$ well geometry parameters.

The asymptotic normalisation constant (ANC) [17] (for large values of $\mathrm{R}$ where it reaches its asymptotic value) was then deduced and the obtained value $\tilde{\mathrm{C}}^{2}=4.5 \pm 2.2 \mathrm{fm}^{-1}$ is found five times larger than that measured by Johnson et al. [10]. Note that the evaluated $49 \%$ uncertainty on $\tilde{\mathrm{C}}^{2}$ is larger than the one given for $\mathrm{S}_{\alpha}$ because it takes into account the dependance of the wave function $\phi(\mathrm{R})$ on the well parameters. However, the evaluated ANC was found nearly independent of the number of nodes $\mathrm{N}$. The above statements are also true for the $\alpha$-reduced width $13.5 \pm 6.6 \mathrm{keV}$ deduced by using the expression $\gamma_{\alpha}^{2}=\frac{\hbar^{2} R}{2 \mu} S_{\alpha}|\phi(R)|^{2}[15]$, where $\mu$ is the reduced mass and $\phi(R)$ is the radial part of the $\alpha-{ }^{13} \mathrm{C}$ cluster wave function calculated at $\mathrm{R}=7.5 \mathrm{fm}$. This radius was chosen in order to reach the coulomb asymptotic behavior of the radial part of the $\alpha_{-}{ }^{13} \mathrm{C}$ wave function.

The contribution of the $1 / 2^{+}$state to the astrophysical S-factor when using our deduced $\gamma_{\alpha}^{2}$ is shown in dashed curve in Figure 3. Its value at $190 \mathrm{keV}$ is $9.6 \times 10^{5} \mathrm{MeV}-\mathrm{b}$, which is five times larger than in reference [10]. The present value for $\gamma_{\alpha}^{2}(6.356 \mathrm{MeV})$ has been used to evaluate the ${ }^{13} \mathrm{C}(\alpha, \mathrm{n}){ }^{16} \mathrm{O}$ S-factor at astrophysical energies. All ${ }^{17} \mathrm{O}$ resonances up to $7.38 \mathrm{MeV}$ have been included in an $R$-matrix calculation. As the resonant structure near the $3 / 2^{+}$resonance at 0.84 $\mathrm{MeV}$ is not well reproduced when using Tilley's resonance parameters [18], we have readjusted the partial widths of this resonance. We find $\Gamma_{\alpha}=0.09 \mathrm{keV}$, and $\Gamma_{n}=400 \mathrm{keV}$. These results are slightly different from the recommended values [18] $\left(\Gamma_{\alpha}=0.07 \mathrm{keV}, \Gamma_{n}=280 \mathrm{keV}\right)$, but are consistent with a ${ }^{16} \mathrm{O}+\mathrm{n}$ measurement of Lister et al. [19]. Note, that in Ref. [10] this problem has been addressed by including a constant term of $0.4 \pm 0.2 \times 10^{6} \mathrm{MeV}-\mathrm{b}$, expected to simulate a nonresonant contribution in the $S$-factor. Our resulting $S$-factor is shown in Fig.3 as a solid curve, with the uncertainty associated with the error bar on $\gamma_{\alpha}^{2}(6.356 \mathrm{MeV})$. At the energy of astrophysical interest, $E_{c m}=0.19 \mathrm{MeV}$, the contribution of the $1 / 2^{+}$subthreshold state to the total S-factor is dominant $(\approx 70 \%)$.

Our calculated ${ }^{13} \mathrm{C}(\alpha, \mathrm{n}){ }^{16} \mathrm{O}$ reaction rate, at temperature $\mathrm{T}=0.09 \mathrm{GK}$ important for the $\mathrm{s}$ process in low mass AGB stars, is found to be 1.3 times less than adopted in NACRE compilation 


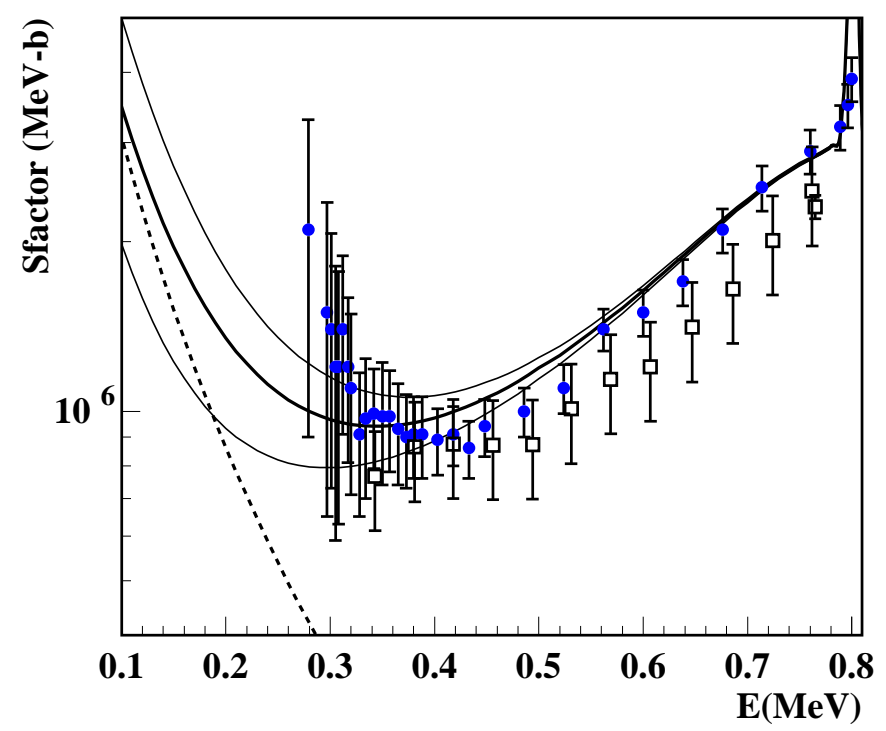

Figure 3: Astrophysical $S$-factor for the ${ }^{13} \mathrm{C}(\alpha, \mathrm{n}){ }^{16}$ reaction with $R$-matrix calculations. The data are taken from Refs.[4, 20]. The contribution of the $6.356 \mathrm{MeV}$ state is shown as the dashed curve. The thick curve correspond to our recommended $\gamma_{\alpha}^{2}$ values, and the thin curves to the lower and upper limits.

and 2.3 times larger than Johnson's deduced rate; $5.41_{-1.8}^{+1.8}, 6.99_{-4.96}^{+1.46}$ and $2.41_{-0.50}^{+0.60} \times 10^{-15} \mathrm{~cm}^{3}$ $\mathrm{mol}^{-1} \mathrm{~s}^{-1}$ respectively.

The discrepancy of a factor as large as 5 between our $1 / 2^{+}$contribution to the reaction rate and the one of Ref. [10] is found reduced to a factor of about 2.3 in the total reaction rate because of the questionnable role of the non-resonant term which was found to be dominant in Ref. [10]. After our new precise determination of the $1 / 2^{+}$state contribution to ${ }^{13} \mathrm{C}(\alpha, \mathrm{n}){ }^{16}$ reaction rate, the non-resonant term is now the main source of error in the evaluation of the reaction rate. This points out the importance to determine experimentally the non-resonant contribution from the angular distributions of ${ }^{13} \mathrm{C}(\alpha, \mathrm{n}){ }^{16} \mathrm{O}$.

\section{Conclusion}

In summary, the reaction ${ }^{13} \mathrm{C}(\alpha, \mathrm{n}){ }^{16} \mathrm{O}$ was investigated through the direct $\alpha$ transfer reaction $\left({ }^{7} \mathrm{Li}, \mathrm{t}\right)$ at 28 and $34 \mathrm{MeV}$ incident energies. The spectroscopic factor and hence the $\alpha$-width and the ANC of the ${ }^{17} \mathrm{O}$ subthreshold state at $6.356 \mathrm{MeV}$ was deduced from a DWBA analysis using different sets of selected optical and well parameters. These parameters were selected in order to fit the measured angular distributions of the various populated states at both incident energies. This procedure leads to a good evaluation of the uncertainties on $S_{\alpha}$ and the $\alpha$-reduced width. The $\alpha$-reduced width was used in the calculation of the astrophysical S-factor and the ${ }^{13} \mathrm{C}(\alpha, \mathrm{n}){ }^{16} \mathrm{O}$ reaction rate at stellar temperatures. The latter was found 2 times larger than the one given in ref [10] and 1.3 times lower than the NACRE adopted value but with a smaller range of allowed values. With our precise measurement [21], the contribution of the $1 / 2^{+}$state is found dominant at astrophysical energies like Keeley's conclusion and contrary to Kubono's and Johnson's results. 


\section{References}

[1] R. Gallino et al., Astrophys. J. 497 (1998) 388

[2] M. Busso et al., Annu . Rev. Astron. Astrophys. 37 (1999) 239

[3] W. Aoki et al., Astrophys. J. Lett. 536 (2000) 197

[4] H. W. Drotleff et al., Astrophys. J. 414 (1993) 735

[5] P. Descouvemont, Phys. Rev. C. 36 (1987) 2206

[6] G. M. Hale, Nuclear physics A 621, 177 (1997)

[7] C. Angulo et al., Nucl. Phys. A 621 (1997) 177c

[8] S. Kubono et al., Phys. Rev. lett. 90 (2003) 062501

[9] N. Keeley et al., Nuclear physics A 726 (2003) 159

[10] E. D. Johnson et al., Phys. Rev. Lett. 97, 192701 (2006)

[11] M. E. Clark, K. W. Kemper, and J. D. Fox Phys. Rev. C 18, 1262 (1978)

[12] I.J. Thomson et al., Comp. Phys. Rep. 7 (1988) 167

[13] J. D. Garrett et al., Nucl. Phys. A 212, 600 (1973)

[14] P. Schumacher et al., Nucl. Phys. A 212, 573 (1973)

[15] F. D. Becchetti et al., Nucl. Phys. A 305 (1978) 293

[16] H. Furutani et al., Prog. Theor. Phys. Suppl. 68, 193 (1980)

[17] A. M. Mukhamedzhanov and R. E. Tribble, Phys. Rev. C. 59, 3418 (1999)

[18] D. R. Tilley et al., Nucl. Phys. A564, 1 (1993)

[19] D. Lister et al., Phys. Rev. 143, 745 (1966)

[20] C. R. Brune, I. Licot, and R. W. Kavanagh, Phys. Rev. C 48, 3119 (1993)

[21] M. G. Pellegriti, F. Hammache et al., Phys.Rev. C 77 (R), 042801 (2008) 